\title{
Kimberlite Pipes from Impacts of Dark Matter being (Ordinary) Matter Compactified into Bubles before BBN
}

\section{Holger Frits Bech Nielsen*}

Niels Bohr Institute, Blegdamsvej 15 -21, DK 2100 Copenhagen

E-mail: hbech@nbi.dk

\section{Mainly with: Colin D. Froggatt}

Glasgow University

E-mail: c.froggattephysics.gla.ac.uk

\begin{abstract}
A certain type of extinct volcanoes called Kimberlite pipes[27] have been suggested by Colin Froaggatt and myself[2] as well as by Mariusz Paszkowski and Jerzy W. Mietelski[1] to be the result of the impact of some "mysterious" material of some type invented by high energy physicists, such as strangelets [49], or our own new phase etc. . We estimate crudely the needed energy to produce a kimberlite pipe volcanoe as well as the number of them having appeared on earth. The density of the material in the outer space needed for the production of the kimberlite pipes is in this way estimated to be not far off order-of-magnitudewise from the density of dark matter in the galaxy arround the solar system as estimated astronomically.
\end{abstract}

Proceedings of the Corfu Summer Institute 2015 "School and Workshops on Elementary Particle Physics and Gravity"

1-27 September 2015

Corfu, Greece

\footnotetext{
* Speaker.
} 


\section{Heading}

\section{Did Dark Matter Cause the Kimberlite Pipes[27] (a strange type of volcanos)?}

I want to be as general as possible by not committing ourselves to too special models, but rather just consider some type of weakly interacting objects of a general mass, and then remark that they could cause a slightly mysterious geological phenomenon, "kimberlite pipes", a kind of volcanos[27] with a little round lake and in the tube such stone as Kimberlite containg diamonds. But of course the present talk is strongly inspired by the work by Colin Froggatt and myself [2].

\section{Introduction}

That the idea, that the Kimberlite pipe volcanoes[27] should have been caused by some impact from outer space, is not completely crazy might be evidenced from that it not only C.D.Froggatt and me[2], who have this thought, but also Paszkowski and Mietelski[1], who consider this possiblity and find it viable.

\section{Review of M. Paszkowski, J.W. Mietelski's work on Kimberlite Pipes}

Vol. 44 (2013) ACTA PHYSICA POLONICA B No 4 p 787:

"ARE KIMBERLITE PIPES A KIND OF MACROSCOPIC NUCLEAR TRACKS FORMED IN COLLISION WITH CUDO?"

Mariusz Paszkowski

Institute of Geological Sciences, Polish Academy of Sciences Senacka 1, 31-002 KrakÃşw, Poland and Poland Jerzy W. Mietelski

The Henryk NiewodniczaÅĎski Institute of Nuclear Physics Polish Academy of Sciences Radzikowskiego 152, 31-342 KrakÃşw, Poland (Received March 8, 2013; revised version received March 26, 2013) [1]

(CUDO=Cosmic Ultra-Dence Objects)

Plan for "Kimberlite Pipes from Impacts of Dark Matter being (Ordinary) Matter Compactified into Bubles before BBN"

- Main part:Mariusz Paszkowski and Jerzy W. Mietelski proposed that the extict volcanos "Kimberlite pipes" were caused by some high energy physics stuff $\approx$ Dark Matter.

- Newest: We estimate the amount of energy and thus Mass needed for a kimberlite pipe production. And what dark matter density?

- Our ideas about:The production of such "dark Matter" as bubles of a different phase in the time before BBN (=Bib Bang Nuclear Synthesis) 


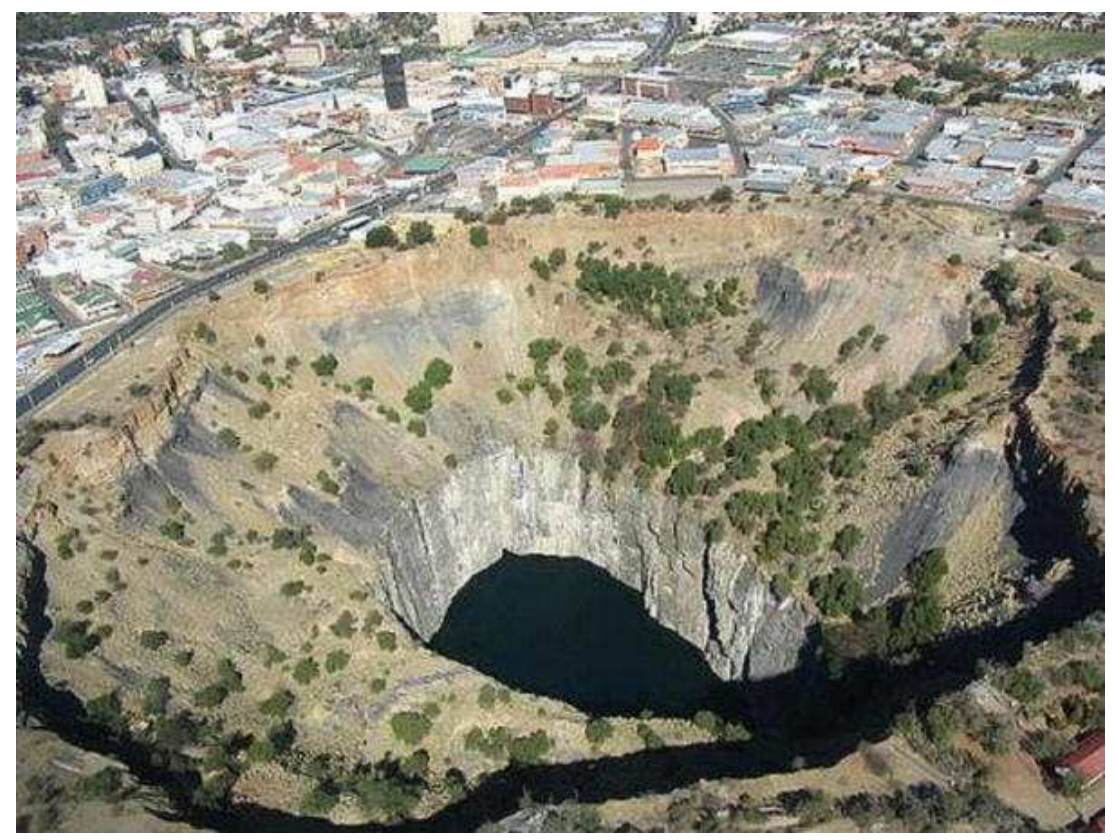

On the figure here you see a big hole a round a lake characteristic of a kimberlite pipe volcanoe[27].

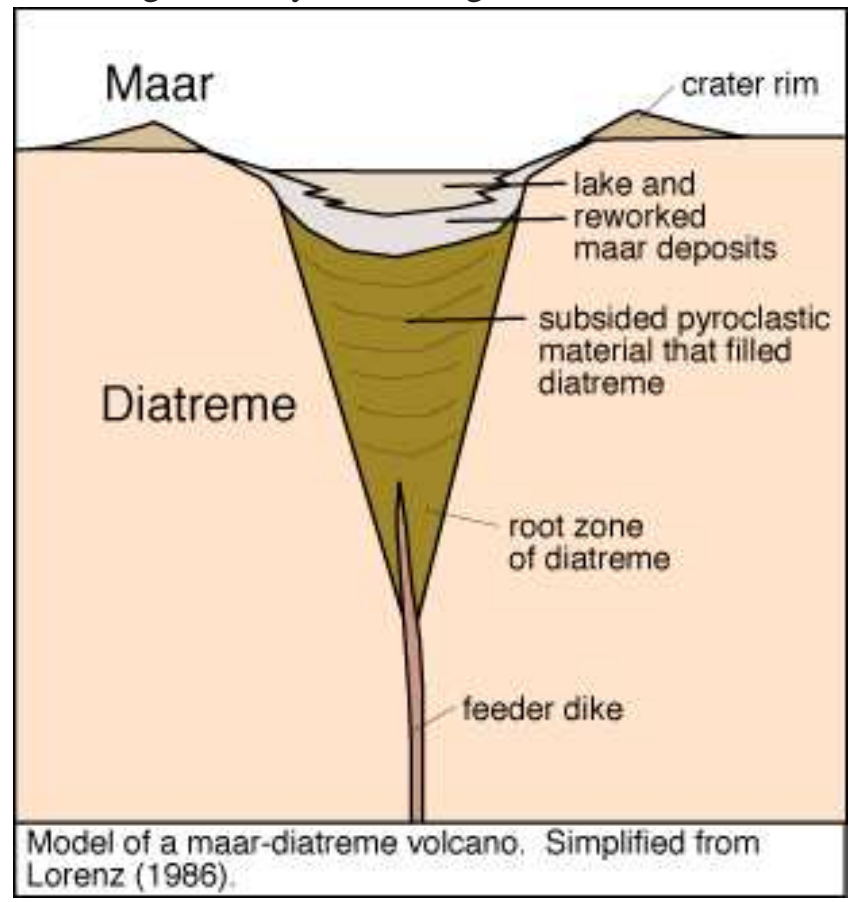

Here above is a more symbolic drawing of a kimberlite pipe volcanoe. 


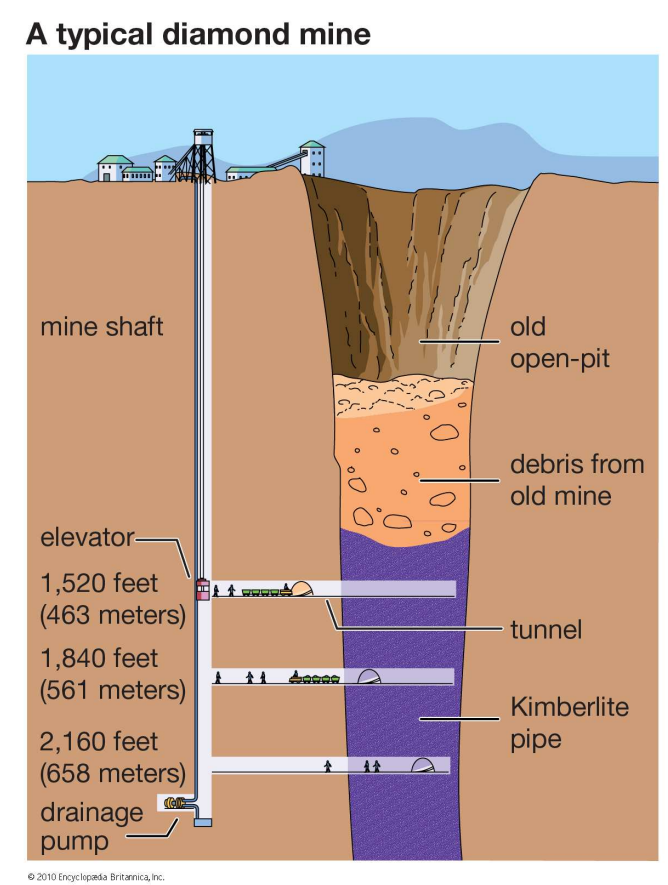

And here one sees, that such pipe is used for diamond mining.

There is large hole in the scale of masses[47] where one tends to forget to look for dark matter particle mass:

between

- The largest mass producable by LHC, say $10 T e V \approx 10^{-20} \mathrm{~g}=10^{-23} \mathrm{~kg}$, and

- the smallest mass visible by gravitational lensing[50] - when passing in th way for the light from behind - of the order of $1 / 3$ moon mass $\approx 2 * 10^{22} \mathrm{~kg}$

hopes have been mainly black holes and interest relatively little.

We shall find by Kimberlite pipes a mass $m \approx 3 * 10^{8} \mathrm{~kg}$ ! Have in mind that the mass of the single dark matter particle has no influence on the large scale flow behavior and gravitational effects; only the dark matter density matters at the large scales of length where the observation astronomically take place

\section{Estimate}

\section{Estimate, Dark matter Energy Matches that Needed for Kimberlite Pipes}

A lower bound for making a kimberlite pipe would seem to be that there should be at least enough energy to lift the material from the mantle/the deep(below the crust) up to nearer to the earth surface. Some energy delivered must be lost by being conducted as heat out into the rock. But still 
we may take say 5 times the energy for just lifting the rocks from the deep to an estimate for what it costs in enrgy to produce the kimberlite pipe.

The crucial question is now:

Does the energy required for making the kimberlite pipes formed during the history of the earth correspond to the amount of energy brought as kinetic energy of dark matter[23, ?]

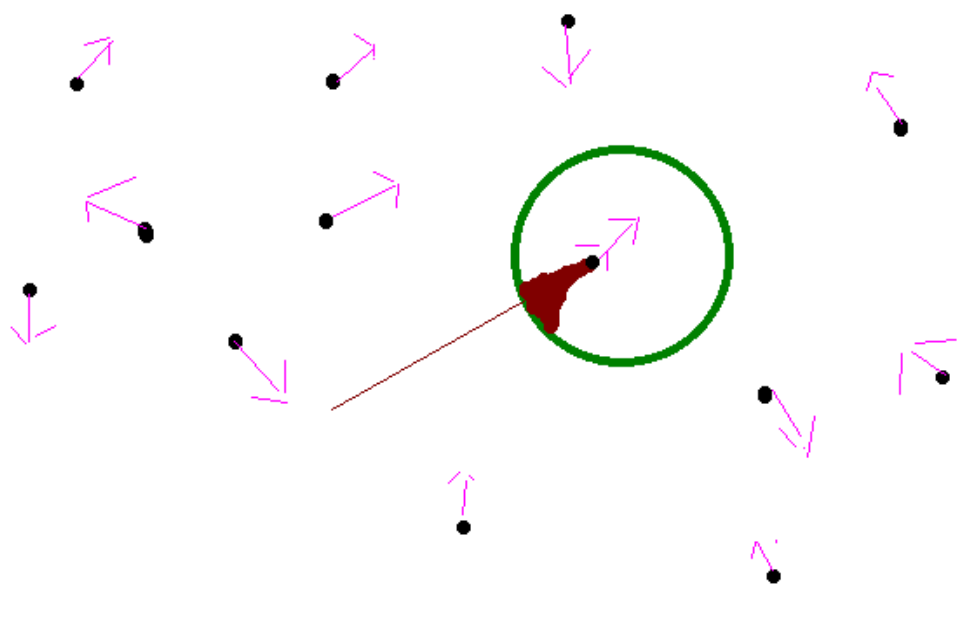

in the corresponding time?

\section{Some Numbers from which to Calculate Dark Matter Density given from Kimberlite Pipes}

The area of Canada:

$$
A=9.984 .670 \mathrm{~km}^{2} \approx 10^{13} \mathrm{~m}^{2},
$$

Number of clusters kimberlite pipes found/exposed in Canada:

$$
c=\# \text { clusters Canada }=30,
$$

The by Colin Froggatt and me estimated probability for a kimberlite pipe (or a cluster of them) to be by accident not covered by sediments, so that it can be found - this is a very crude estimate, to which we may return-:

$$
P=P(\text { exposure }) \approx 2.2 * 10^{-3} ;
$$

\section{More Numbers from which to Estimate}

Existence or life time of the earth - time during which the impacts of CUDOs may have taken place:

$$
l=\text { "earth life time" } \approx 4 * 10^{9} \text { years }=31536000 s * 4 * 10^{9} \approx 1.2 * 10^{17} s,
$$

Typical diameter of the deep part of the kimberlite pipe

$$
d \approx \text { "a few to a dozen } \mathrm{m} " \approx 5 \mathrm{~m},
$$

Hight of the earth suface over "some place deep in the mantle"

$$
h \geq 200 \mathrm{~km}=2 * 10^{5} \mathrm{msay}: h \approx 500 \mathrm{~km}=5 * 10^{5} \mathrm{~m},
$$

Earth gravitational acceleration:

$$
g=9.8 m / s^{2} \approx 10 m / s^{2}
$$




\section{Yet some Numbers for Estimate}

Density of the material making up the crust and mantle of earth which is to be replaced by the kimberlite in the impact happening:

$$
\rho_{\text {stone }} \approx 3 \mathrm{~g} / \mathrm{cm}^{3}=3000 \mathrm{~kg} / \mathrm{m}^{3}
$$

Velocity relative to the earth of a dark matter object from the halo or, that has been caught by the solar system, wiehgted crudely with the relative probability of these two possibilities:

$$
v=200 \mathrm{~km} / \mathrm{s}=2 * 10^{5} \mathrm{~m} / \mathrm{s}
$$

Let us also introduce a symbol $\zeta$ for the fraction of the energy of an impact CUDO being in reality used for the very pumping up of the kimberlite-material from the mantle defth to closer at at the surface of the earth, say:

$$
\zeta \approx 0.2=1 / 5
$$

\section{Estimating the Mass of Dark Matter Ball / CUDO in Two ways}

First we estimate -very crudely -the mass $m$ (called then $\left.m\right|_{\text {pipe }}$ ) from the energy needed to lead to the pumping up from the mantle defth - say $h=500 \mathrm{~km}$ - of the material - the kimberlite material forming a pipe of diameter of the order of $d=5 m$ say:

$$
\begin{aligned}
\left.m\right|_{\text {pipe }} & =\frac{E}{\zeta * 1 / 2 * v^{2}}(\text { where } E \text { is “uppumping energy") } \\
& =\frac{\rho_{\text {stone } * g * h^{2} * d^{2}}}{\zeta * 1 / 2 * v^{2}} \\
& =\frac{3 * 10^{3} \mathrm{~kg} / \mathrm{m}^{3} * 10 \mathrm{~m} / \mathrm{s}^{2} *\left(5 * 10^{5} \mathrm{~m}\right)^{2} *(5 \mathrm{~m})^{2}}{1 / 5 * 1 / 2 *\left(2 * 10^{5} \mathrm{~m} / \mathrm{s}\right)^{2}} \\
& =\frac{375 * 10^{14} \mathrm{~kg}}{4 / 10 * 10^{10}} \\
& \approx 10^{7} \mathrm{~kg}=10^{4} \mathrm{ton}
\end{aligned}
$$

\section{Estimating the Mass of Dark Matter Ball / CUDO in Two ways}

Next we estimate the same dark matter ball mass $m$ from the density of dark matter in the solar neighborhood taken to be $\rho_{D M}=1 \mathrm{GeV} / \mathrm{cm}^{3}$ as supposed from astronomy, now called $\left.m\right|_{\text {DMdensity }}$,

$$
\begin{gathered}
\left.m\right|_{D M d e n s i t y}= \\
=\frac{\rho_{D M} l v \frac{1}{4} A}{1 / P * c} \\
=\frac{1.7 * 10^{-21} \mathrm{~kg} / \mathrm{m}^{3} * 10^{17} \mathrm{~s} * 2 * 10^{5} \mathrm{~m} * 1 / 4 * 10^{13} \mathrm{~m}^{2} * 2.2 * 10^{-3}}{30} \\
=0.6 * 10^{10} \mathrm{~kg}=6 * 10^{9} \mathrm{~kg} \\
=6 * 10^{6} \text { ton }
\end{gathered}
$$




\section{Slightly Better Estimate of the Corpuscle Masses $m_{\text {pipe }}$ and $m_{D M d e n s i t y}$.}

The kimberlite pipe is seen to broaden out near the top where it passes the earth surface to a diameter of "several hundred meters", so we should rather use the diameter to be $d \approx 500 \mathrm{~m}$ than the diameter $d \approx 5$, which is more relevant for the deep inside earth part of the kimberlite pipe, and that would increase the estimate of the volume of material lifted by a factor $(500 / 5)^{2}=10000$. But then we would have to take into account that the broad part of the kimberlite pipe only goes down about $1.5 \mathrm{~km}$ to $2 \mathrm{~km}$, thus we should replace the factor $h=5 * 10^{5} \mathrm{~m}$ by only $1.5 \mathrm{~km}=1.5 * 10^{3} \mathrm{~m}$, which is 300 times smaller. By this correction we would get

$$
m_{\text {pipe }} \approx 3 * 10^{8} \mathrm{~kg}=3 * 10^{5} \text { ton }
$$

We indeed estimate with hight of the thick part of the pipe $h^{\prime} \approx 1.5 \mathrm{~km}$, while material is pumped from the defth $h \approx 500 \mathrm{~km}$ and diameter of this thick part being $d \approx 500 \mathrm{~m}$ :

$$
\begin{aligned}
& m_{\text {pipe }} \\
& =\frac{\rho_{\text {stone }} * g * h^{\prime} h * d^{2}}{\zeta * 1 / 2 * v^{2}} \\
& =\frac{3 * 10^{3} \mathrm{~kg} / \mathrm{m}^{3} * 10 \mathrm{~m} / \mathrm{s}^{2} *\left(1.5 * 10^{3} * 5 * 10^{5} \mathrm{~m}\right) *(500 \mathrm{~m})^{2}}{1 / 5 * 1 / 2 *\left(2 * 10^{5} \mathrm{~m} / \mathrm{s}\right)^{2}} \\
& \approx 3 * 10^{8} \mathrm{~kg}=3 * 10^{5} \text { ton }
\end{aligned}
$$

\section{Nearer Thinking of Estimating the Mass $m_{D M d e n s i t y}$ from Astronomically Known Dark Matter Density.}

On the whole earth it were claimed that $c_{\text {earth }}=6400$ kimberlite pipes had been found(rather than our number $c=30$ for Canada, which has an area 50 times smaller). Including a factor $\frac{1}{4}$ for the probability of the area being turned in the right direction for being hit as if perpendicularly the quarter of the surface area is $\frac{1}{4} A_{\text {earth }}=\pi R_{\text {earth }}^{2}=1.275 * 10^{14} \mathrm{~m}^{2}$ (to compare with Canadas $\frac{1}{4} A=$ $\frac{1}{4} 10^{13} \mathrm{~m}^{2}$ ). If we should only expect that one found these Kimberlite pipes on land, then we replace this number by $29 \%$ of it. In that case we would effectively have $\frac{1}{4} A$ replaced by $\frac{1}{4} A_{\text {land }}=.29 *$ $1.28 * 10^{14} \mathrm{~m}^{2}=3.7 * 10^{13} \mathrm{~m}^{2}$ and the mass of the dark matter particle would have to be:

$$
\begin{aligned}
& m_{\text {DMdensity }} \approx 4 * 10^{5} \text { ton }, \\
& m_{\text {DMdensity }} \approx 4 * 10^{5} \text { ton, }
\end{aligned}
$$

means that matches phantastically well with the from the size and defth of Kimberlite pipes estimated $m_{\text {pipe }} \approx 3 * 10^{5}$ ton:

$$
m_{\text {pipe }} \approx m_{D M \text { density }},
$$

or it means that we from studies of the Kimberlite pipes in number and requirement energy would have been able to predict the dark matter density to: $\left.\rho_{D M}\right|_{\text {from Kimberlite pipes }} \approx 3 / 4 * 1 \mathrm{GeV} / \mathrm{cm}^{3} \approx$ $.7_{5} \mathrm{GeV} / \mathrm{cm}^{3}$. So we really fit well to all dark matter being the material causing the Kimberlite pipes in the earth! The calculation of the mass of a single dark matter corpuscle mass $m_{D M d e n s i t y}$ 
in order to fit the astronomically needed dark matter density $\rho D M=1.7 * 10^{-21} \mathrm{~kg} / \mathrm{m}^{3}$ - and a velocity $v=200 \mathrm{~km} / \mathrm{s}$ - with the number of kimberlite pipes gives:

$$
\begin{aligned}
\left.m\right|_{\text {DMdensity }}= & \\
& =\frac{\rho_{D M} l v \frac{1}{4} A}{1 / P * c} \\
& =\frac{1.7 * 10^{-21} \mathrm{~kg} / \mathrm{m}^{3} * 10^{17} \mathrm{~s} * 2 * 10^{5} \mathrm{~m} / \mathrm{s} * 3.7 * 10^{13} \mathrm{~m}^{2} * 2.2 * 10^{-3}}{6400} \\
& =4 * 10^{8} \mathrm{~kg}=4 * 10^{5} \mathrm{ton}
\end{aligned}
$$

\section{Conclusion Concerning the Posibility of the Kimberlite Pipes found on Earth could be Dark Matter Caused}

- Within our very crude estimates the energy density astronomically found for dark matter fits well with the amount of energy estimated to be needed to produce the kimberlite pipes found geologically on earth(assuming a typical halo velocity $200 \mathrm{~km} / \mathrm{s}$ for the speed of the objects).

- The mass of the individuel dark matter particles associated with this fit is $m_{D M d e n s i t y} \approx$ $m_{\text {pipe }} \approx(3$ to 4$) * 10^{5}$ ton.

- The average time interval between impacts of dark matter on land is

$$
\begin{aligned}
& \left.\Delta t\right|_{\text {impact }} \\
= & \frac{m}{\rho_{D M} v \frac{1}{4} A_{\text {earth }} * .29} \\
= & \frac{3 * 10^{11} \mathrm{~kg}}{1.3 * 10^{-17} \mathrm{~kg} / \mathrm{m}^{3} * 2 * 10^{5} \mathrm{~m} / \mathrm{s} 1.28 * 10^{14} * .29} \\
= & 3 * 10^{9} \mathrm{~s}=100 \text { years. }
\end{aligned}
$$

(Just the time since the Tunguska event[3, 28, 29, 30, ?] in 1908)

- Typical distance between neighboring dark matter balls/particles/ corpuscles in the earth region

$$
\begin{aligned}
& \text { dist }_{\text {DM particles }} \\
= & \left(\frac{\mathrm{m}}{\rho_{D M}}\right)^{1 / 3} \\
= & \left(\frac{3 * 10^{11} \mathrm{~kg}}{1.3 * 10^{-17} \mathrm{~kg} / \mathrm{m}^{3}}\right)^{1 / 3} \\
= & 3 * 10^{9} \mathrm{~m}=10^{-7} \mathrm{pc}=2 * 10^{-2} \text { a.u. }
\end{aligned}
$$


Even if it were of the maximal size if the pipe should be only say $5 \mathrm{~m}$ in diameter meaning 5 $\mathrm{m}$, then it would be 700000 times smaller than the moon - which has -12.6th magnitude, but 10 times longer away, so it would be of of 21 st magnitude most optimisticly. But more like as in our more concrete model of $\mathrm{cm}$-size pearls they have 28th to 29th magnitude. Hubble could (goes to 30th magnitude[48])in the deepest field search, but could not find it.

\section{Big Bang Time Production of Machroscopic WIMPS}

\section{What Could Such Dark Matter in Clumps of $3 * 10^{5}$ Ton be?}

- Presumably composite objects of many particles held strongly together somehow ?!

- "New Physics Stuff" or made from usual baryons electrons etc.?

- If usual baryons etc., it must be packed in a form unaccessible to the $\mathrm{BBN}(=\mathrm{Big}$ Bang Nuclear Synthesis) processes; otherwise the predictions from BBN of light isotope abundances would be disturbed.

\section{Generally Such New Matter Phases must be Isolated from BBN to not Disturb}

Both quark nuggetts and strangeletts are made from quarks - meaning at the end ordinary matter - so they should be already in a form before say the Universe of the order of 1/10 to 1/100 s old have come into a form that does not interact (normally) with the other nucleons in the era of the Big Bang Nuclear synthesis, because otherwise the production of the abundances of light elements such as helium and lithium and isotopes deuterium etc. would not fit to give $\eta$ - meaning the ratio of baryons to photons - matching with only the normal matter, but rather a bigger $\eta$-value.

\section{Our own Model of a 6 top +6 anti top Bound State Phase}

Looking very abstractly on it there is not much difference between the quark-nuggetts, the strangeletts, and our own picture with a new type of vacuum characterized by a boson condensate of a speculated very strongly bound state of 6 top +6 anti-top quarks a buble of which is then filled by very compressed ordinary matter:

All these models mean that the dark matter consists a quarks( $\approx$ ordinary matter $)$ in a very condenced phase.

Then it can

- 1. Be kept so strongly bound that it does not interfere in BBN.

- 2. and that it does neither interact much with gas in the gallaxy (when have the huge clumps the quarks or nucleons hides each other from the interactions with light and matter; only the relatively few surface quarks may interact.

\section{A Problem ?:}


One can presumably tune the parameters, but would it not be more likely if the to-be-dark-matter phase is formed to get almost all matter made into dark matter before the BBN ?

We[2] - C. D. Froggatt and I - has it basically all got into the dark matter pearls; but then the helium inside the pearls fusion to heavier elements and by the heat produced nucleons are expelled to become the ordinary matter.

\section{Conclusion}

- The very thin piped volcano type called Kimberlite pipes - which are a bit of a mystery - can be explained as due to dark matter impact.

- The estimated density of dark matter needed to fit the Kimberlite pipes agrees crudely remarkably well with the order of magnitude of dark matter density observed astronimically by its gravitation!

- The proposals such as quark nuggetts, strangeletts, or our own bound state condensate with ordinary matter filling are all basically new/speculated phases of ordinary matter.

- The new phase has to be "finished" before BBN, but that is possible ...

- In our own model a fusion explosion predicts a very good ratio of dark to normal matter.

Picture of the Estimates

\begin{tabular}{|c|c|c|}
\hline Kimberlite pipe Energy & $\frac{1}{2} v^{2} * m_{\text {pipe }}$ & $6 * 10^{18} \mathrm{~J}=6 \mathrm{EJ}$ \\
Dark Pearl Mass & $m_{\text {pipe }} \approx m_{D M \text { density }}$ & $3 * 10^{8} \mathrm{~kg}=3 * 10^{5}$ ton \\
Hit earth $\sim$ every & & 100 years \\
\hline
\end{tabular}

\section{References}

[1] Mariusz Paszkowski, Institute of Geological Sciences, Polish Academy of Sciences Senacka 1, 31-002 KrakÃşw, Poland and Jerzy W. Mietelski, The Henryk NiewodniczaÅĎski Institute of Nuclear Physics Polish Academy of Sciences Radzikowskiego 152, 31-342 KrakÃşw, Poland (Received March 8, 2013; revised version received March 26, 2013) “ARE KIMBERLITE PIPES A KIND OF MACROSCOPIC NUCLEAR TRACKS FORMED IN COLLISION WITH CUDO?” Vol. 44 (2013) ACTA PHYSICA POLONICA B No 4 p 787:

(CUDO=Cosmic Ultra-Dence Objects)

[2] C. D. Froggatt and H. B. Nielsen, “Tunguska Dark Matter Ball,” Int. J. Mod. Phys. A 30 (2015) no.13, 1550066 doi:10.1142/S0217751X15500669 [arXiv:1403.7177 [hep-ph]].

[3] G. Longo, The Tunguska event. In: Comet/Asteroid Impacts and Human Societ, An Interdisciplinary Approach eds: P. T. Bobrowsky and H. Rickman (Springer-Verlag, 2007) pp 303-330.

[4] L. Casperini, L. Cocchi, C. Stanghellini, G. Del Bianco, M. Serrazanetti and M. Carmisciano, Geochem. Geophys. Geosyst. 13 (2012) Q05008.

[5] Mystery of Tunguska meteorite solved, english.pravda.ru 25.10.2010. 
[6] P. H. Frampton, JCAP 0910 (2009) 016. [arXiv:0905.3632];

P. H. Frampton, M. Kawasaki, F. Takahashi and T. Yanagida, JCAP 1004 (2010) 023 [arXiv:1001.2308].

[7] C. D. Froggatt and H. B. Nielsen, Phys. Rev. Lett. 95 (2005) 231301 [arXiv:astro-ph/0508513]; Proc. of 8th Workshop on What comes beyond the Standard Model, Bled 2005, pp 32-39 [arXiv:astro-ph/051245].

[8] D. H. Oaknin and A. Zhinitsky, Phys. Rev. D71 (2005) 023519 [arXiv:hep-ph/0309086].

[9] D. L. Bennett, C. D. Froggatt and H. B. Nielsen, Proc. of the 27th International Conference on High Energy Physics, edited by P. Bussey and I. Knowles (IOP Publishing Ltd, london, 1995), p. 557; Perspectives in Particle Physics '94, edited by D. Klabučar, I. Picek and D. Tadic̀ (World Scientific, Singapore, 1995), p. 255 [arXiv:hep-ph/9504294];

D. L. Bennett and H. B. Nielsen, Int. J. Mod. Phys. A9 (1994) 5155.

[10] C. D. Froggatt and H. B. Nielsen, Phys. Lett. B368 (1996) 96 [arXiv:hep-ph/9511371].

[11] N. Cabibbo, L. Maiani, G. Parisi and R. Petronzio, Nucl. Phys. B158 (1979) 295;

M. Lindner, Z. Phys. C31 (1986) 295;

M. Lindner, M. Sher and H.W. Zaglauer, Phys. Lett. B228 (1989) 139.

[12] F. Bezrukov, M. Y. Kalmykov, B. A. Kniehl and M. Shaposhnikov, JHEP 1210 (2012) 140 [arXiv:1205.2893];

G. Degrassi, S. Di Vita, J. Elias-Miro, J. R. Espinosa, G. F. Giudice, G. Isidori and A. Strumia, JHEP 08 (2012) 098 [arXiv:1205.6497];

D. Buttazzo, G. Degrassi, P. P. Giardino, G. F. Giudice, F. Sala, A. Salvio and A. Strumia, JHEP 12 (2013) 089 [arXiv:1307.3536].

[13] CDF, D0 Collaborations, Phys. Rev. D86 (2012) 092003 [arXiv:1207.1069]; ATLAS Collaboration, CMS Collaboration, S. Blywert, arXiv:1205.2175.

[14] S. Alekhin, A. Dijouadi and S. Moch, Phys. Lett. B716 (2012) 214 [arXiv:1207.0980].

[15] ATLAS Collaboration, Phys. Lett. B716 (2012) 1 [arXiv:1207.7214];

CMS Collaboration, Phys. Lett. B716 (2012) 30 [arXiv:1207.7235];

CMS Collaboration, CMS PAS HIG-12-045.

[16] C. D. Froggatt, H. B. Nielsen and Y. Takanishi Phys. Rev. D64 (2001) 113014 [arXiv:hep-ph/0104161].

[17] J. Elias-MirÃşa, J. R. Espinosa, G. F. Giudice, G. Isidori, A. Riotto and A. Strumia, Phys. Lett. B709 (2012) 222 [arXiv:1112.3022].

[18] C. D. Froggatt and H. B. Nielsen, Surv. High Energy Phys. 18 (2003) 55 [arXiv:hep-ph/0308144]; Proceedings to the Euroconference on Symmetries Beyond the Standard Model (DMFA, Zaloznistvo, 2003), p.73 [arXiv:hep-ph/0312218];

C. D. Froggatt, H. B. Nielsen and L. V. Laperashvili, Int. J. Mod. Phys. A20 (2005) 1268 [arXiv:hep-ph/0406110].

[19] C. D. Froggatt and H. B. Nielsen, Phys. Rev. D80 (2009) 034033 [arXiv:0811.2089];

[20] M. Yu. Kuchiev, V. V. Flambaum and E. Shuryak, Phys. Rev. D78 (2008) 077502 [arXiv:0808.3632]; J.-M. Richard, Few Body Syst. 45 (2009) 65 [arXiv:0811.2711];

M. Yu. Kuchiev, Phys. Rev. D82 (2010) 127701 [arXiv:1009.2012].

[21] E. W. Kolb and M. S. Turner, The Early Universe (Addison-Wesley, Reading, MA, 1990). 
[22] W. Kundt, Current Science 81 (2001) 399; Chin. J. Astron. Astrophys 3 (2003), Suppl. 545.

[23] M. Kanionkowski and A. Kinkhabwala, Phys. Rev. D57 (1998) 3256.

[24] T. Bruch, J. Read, L. Baudis and G. Lake Astrophys. J. 696 (2009) 920. [arXiv:0806.3767].

[25] K. Nakamura et al. (Particle Data Group), Phys. Rev. D86 (2012) 010001.

[26] V. V. Flambaum and R. B. Wiringa, Phys.Rev. C 76 (2007) 054002 [arXiv:0709.0077].

[27] J. B. Dawson Kimberlites and their xenoliths (Springer-Verlag, 1980);

R. H. Mitchell Kimberlites: Mineralogy, geochemistry and petrology (Plenum Press, 1986).

[28] L. A. Kulik, Doklady AN SSR, seria A 23 (1927) 393.

[29] Tunguska event, en.wikipedia.org/wiki/Tunguska_event.

[30] L. Gasperini, F. Alvisi, G. Biasini, E. Bonatti, G. Longo, M. Pipan, M. Ravaioli and R. Serra, Terra Nova 19 (2007) 245;

G. S. Collins, N. Artemieva, K. Wunnemann, P. A.Bland, W.U.Reimold and C. Koeberl, Terra Nova 20 (2008) 165;

L. Gasperini, E. Bonatti and G. Longo, Terra Nova 202008 169;

L. Gasperini, E. Bonatti, S. Albertazzi, L. Forlani, G. Accorsi, G. Longo, M. Ravaioli, F. Alvisi,

A. Polonia and F. Sacchetti, Terra Nova 21 (2009) 489.

[31] K. L. Rasmussen, H. B. Clausen and G. W. Kallemeyn, Meteoritics 30 (1995) 634;

K. L. Rasmussen, H. J. Olsen, R. Gwozdz and E. M. Kolesnikov, Meteoritics and Planetary Science 34 (1999) 891.

[32] E. M. Kolesnikov, T. Boettger and N. V. Kolesnikova, Planet. Space Sci. 47 (1999) 905.

[33] E. M. Kolesnikov, G. Longo, T. Boettger, N. V. Kolesnikov, P. Gioacchini, L. Forlani, R. Giampieri and R. Serra, Icarus 161 (2003) 235.

[34] P. Deines, Earth Science Reviews 58 (2002) 247.

[35] G. R. Grimmett and D. R. Stirzaker, Probability and Random Processes, (Oxford University Press, 2001)

[36] B. H. Scott Smith, J. Volcanology and Geothermal Research, 174 (2008) 9.

[37] H. Jelsma, W. Barnett, S. Richards and G. Lister, Lithos 112S (2009) 155.

[38] L. M. Heaman, B. A. Kjarsgaard and R. A. Creaser, Lithos 71 (2003) 153; ibid 76 (2004) 377.

[39] Planck Collab., P. A. R. Ade et al, arXiv:1303.5076.

[40] K. A. Olive, G. Steigman and T. P. Walker, Phys. Reports 333 (2000) 389;

F. Iocco, G. Mangano, G. Miele, O. Pisanti and P. D. Serpico, Phys. Reports 472 (2009) 1.

[41] T. Kibble, J. Phys. A9 (1976) 1387.

[42] C. D. Froggatt and H. B. Nielsen, in Proceedings of the 34th International Conference in High Energy Physics (ICHEP08), Philadelphia, 2008, eConf C080730, [hep-ph/0810.0475].

[43] DAMA Collab., R. Bernabei et al, Eur. Phys. J. C67 (2010) 39 [arXiv:1002.1028].

[44] COGENT Collab., C. E. Aalseth et al, Phys. Rev. Lett. 107 (2011) 141301 [arXiv:1106.0650].

[45] CRESST Collab., G. Angloher et al, Eur. Phys. J. C72 (2012) 1971 [arXiv:1109.0702]. 
[46] CDMS and EDELWEISS Collabs., Z. Ahmed et al, Phys. Rev. 84 (2011) 011102 [arXiv:1105.3377]; XENON Collab., E. Aprile et al, Phys. Rev. Lett. 109 (2012) 181301 [arXiv:1207.5988];

LUX Collab., D. S. Akerib et al, arXiv:1310:8214.

[47] MACHO Collab., C. Alcock et al, Astrophys. J. 542 (2000) 257 [arXiv:astro-ph/0001272];

EROS Collab., P. Tisserand et al, A\&A 469 (2007) 387 [arXiv:astro-ph/0607207];

OGLE Collab., L. Wyrzykowski et al, MNRAS 416 (2011) 2949 [arXiv:1106.2925].

[48] https://lcogt.net/spacebook/what-apparent-magnitude/

[49] E. Farhi and R. Jaffe, "Strange Matter", Phys. Rev. D30, 2379 (1984) E. Witten, "Cosmic Separation Of Phases" Phys. Rev. D30, 272 (1984) A. Bodmer "Collapsed Nuclei" Phys. Rev. D4, 1601 (1971) H. Heiselberg, "Screening in quark droplets", Phys. Rev. D48, 1418 (1993) M. Alford, K. Rajagopal, S. Reddy, A. Steiner, "The Stability of Strange Star Crusts and Strangelets", Phys. Rev. D73 114016 (2006) arXiv:hep-ph/0604134 Shibaji Banerjee, Sanjay K. Ghosh, Sibaji Raha, and Debapriyo Syam, "Can Cosmic Strangelets Reach the Earth?", Phys. Rev. Lett. 85, 1384 âĂŞ Published 14 August 2000, http://arxiv.org/abs/hep-ph/0006286 STAR Collaboration, "Strangelet search at RHIC", arXiv:nucl-ex/0511047 Ellis J, Giudice G, Mangano ML, Tkachev I, Wiedemann U (LHC Safety Assessment Group) (5 September 2008). "Review of the Safety of LHC Collisions" (PDF, 586 KiB). "Journal of Physics G: Nuclear and Particle Physics. 35, 115004 (18pp). doi:10.1088/0954-3899/35/11/115004. arXiv:0806.3414. CERN record.

[50] M. Bartelmann and P. Schneider, "Weak gravitational lensing," Phys. Rept. 340 (2001) 291 doi:10.1016/S0370-1573(00)00082-X [astro-ph/9912508]. 